Research Article

\title{
Electromechanical Impedance Analysis on Piezoelectric Smart Beam with a Crack Based on Spectral Element Method
}

\author{
Dansheng Wang, Hongyuan Song, and Hongping Zhu \\ School of Civil Engineering and Mechanics, Huazhong University of Science and Technology, Wuhan 430074, China \\ Correspondence should be addressed to Dansheng Wang; danshwang@hust.edu.cn
}

Received 11 April 2014; Revised 6 July 2014; Accepted 16 July 2014

Academic Editor: Kui Fu Chen

Copyright (c) 2015 Dansheng Wang et al. This is an open access article distributed under the Creative Commons Attribution License, which permits unrestricted use, distribution, and reproduction in any medium, provided the original work is properly cited.

An electromechanical impedance (EMI) analysis of a piezoelectric smart beam with a crack is implemented in this paper. Spectral element method (SEM) is used to analyze the EMI response of the piezoelectric smart beam. In this analysis, the spectral element stiffness matrices of different beam segments are derived in this paper. The crack is simulated using spring models, and the EMI signatures of piezoelectric smart beam with and without crack are calculated using SEM, respectively. From the analysis results, it is found that the peak position and amplitude of the EMI signatures have significant changes with the change in crack depth, especially in higher frequency ranges. Different vibration modes of the piezoelectric smart beam are analyzed, and the effect of thickness of the adhesive layer on the admittance is also researched. An experimental study is also implemented to verify the validity of the analysis results using SEM.

\section{Introduction}

The presence of structural damage will result in the changes in the properties of the structure and will also induce the change in the mechanical impedance of the structure. Damage identification can be achieved by comparing the structural mechanical impedances before and after damage. However, structural mechanical impedance is difficult to measure in practice. The coupled electromechanical impedance (EMI) of structure with PZT patches bonded to it is convenient to obtain by using impedance analyzer, even in the high frequency ranges, which is quite sensitive to structural incipient damage. In recent years, the EMI-based technique has been applied to structural health monitoring. It has been successfully used to analyze various engineering structures, including aerospace structures, mechanical structures, and civil structures [1-11]. The EMI technique is based on the direct and converse piezoelectric effects of the PZT patch. In order to analyze the EMI of coupled structures, the model characterizing electromechanical interaction between the host structure and PZT patch should be firstly established. Liang et al. [12] proposed the first EMI model for a PZT patch bonded onto an intact one-dimensional structure. Zhou et al. [13] extended the impedance method to model a twodimensional EMI structure. Bhalla and Soh also presented an improved 2D impedance model to characterize the PZTstructure electroelastic interactions based on the concept of "effective impedance" [14]. Annamdas and Soh have examined the three-dimensional interaction of a PZT transducer or multiple PZT transducers with the host structure based on directional sum impedance formulation $[15,16]$. Wang et al. also proposed an embedded 3D electromechanical impedance model for an embedded PZT transducer by considering the interaction between a square PZT patch and a host structure [17].

Spectral element method (SEM) has been proposed to the structural dynamics community as a numerical tool to analyze the dynamic responses of rods, beam, and plates [18]. Spectral element is represented by an exponential interpolation function, which is an exact solution of the wave equation for representative structural element. Therefore, other than classical finite element method, SEM provides dynamic stiffness matrix of a structure, which is more accurate and efficient than the traditional static stiffness matrix, 
and allows an increased accuracy in modeling the structural dynamic behavior. Subsequently, Palacz and Krawczuk [19] analyzed the dynamic responses of a cracked rod using SEM. The crack is simulated by a rotational spring model, and the node displacement and shape function of the cracked rod are derived. Ostachowicz et al. further summarized the researches on spectral element method and derived the spectral element stiffness matrices of Euler beam, Timoshenko beam, damaged plate, and thin-walled shell structures [2023]. Samaratunga et al. presented a new $2 \mathrm{D}$ wavelet spectral finite element model for studying wave propagation in thin to moderately thick anisotropic composite laminates [24]. Choi and Inman recently presented modeling of a cableharnessed structure by means of SEM, and a double beam model was formulated. The presented modeling was applied and compared with the conventional FEM to emulate a cableharnessed structure [25].

Some researchers also focused on identifying the damage from the EMI signatures of the cracked beam with surfacebonded PZT patches using SEM. Park et al. implemented damage identification study on a simple one-dimensional structure by combining SEM and EMI techniques [26, 27]. Ritdumrongkul et al. succeeded in quantitative identification of the structure damage based on the SEM and PZT active sensors. In the experiment, a bolted aluminum beam is studied, and the damage is simulated by looseness of the bolt [28]. Wang and Tang further deduced the stiffness matrix of smart Timoshenko beam and successfully analyzed electric admittances of piezoelectric smart beam using the SEM [29]. Combined with EMI method and nonlinear optimization technique, the spectral element model of a simply supported rod was simulated and numerical study on localization and quantitative identification of damage was made by Guo and Sun [30]. Ostachowicz et al. developed their own numerical procedures using SEM in order to calculate damage indexes, which were used both for damage detection and for localization. The proposed methods were applied for structural health monitoring of metallic and fibre reinforced structural elements [31]. Kim and Wang proposed an improved impedance-based damage identification method by incorporating a tunable piezoelectric circuitry with the structure to enrich the impedance measurements. Numerical case study on localizing damage in a fixed-fixed beam using SEM was performed to demonstrate the effectiveness of the new method for structural damage identification [32].

In this paper, a new spectral element model of a cracked Timoshenko beam is proposed. In the model, the crack is modeled accurately by three massless springs, that is, a shear spring, a translational spring, and a rotational spring. According to the relationship between dynamic load and displacement, the spectral element stiffness matrices of different beam segments are obtained, and then the EMI of the piezoelectric coupled beam with a crack is formulized. Subsequently, the proposed spectral element model is validated by experimental results. Finally, numerical investigations are addressed to analyze the EMI responses of the piezoelectric Timoshenko beam with a crack based on the spectral element model.

\section{Spectral Element Formulations}

2.1. Spectral Element Stiffness Matrix of an Intact Timoshenko Beam. The governing differential equations for a Timoshenko beam are given as

$$
\begin{gathered}
\kappa G A\left(\frac{\partial^{2} w}{\partial x^{2}}-\frac{\partial \varphi}{\partial x}\right)=\rho A \frac{\partial^{2} w}{\partial t^{2}}, \\
E I \frac{\partial^{2} \varphi}{\partial x^{2}}+\kappa G A\left(\frac{\partial w}{\partial x}-\varphi\right)=\rho I \frac{\partial^{2} \varphi}{\partial t^{2}}, \\
E \frac{\partial^{2} u}{\partial x^{2}}-\rho \frac{\partial^{2} u}{\partial t^{2}}=0, \\
Q=\kappa G A\left(\frac{\partial w}{\partial x}-\varphi\right), \quad M=E I \frac{\partial \varphi}{\partial x}, \\
N=E A \frac{\partial u}{\partial x},
\end{gathered}
$$

where $u, w$, and $\varphi$ are the axial, rotational, and shear displacements, respectively. $N, Q$, and $M$ denote the axial force, transverse force, and bending moment, respectively. $E$ is Young's modulus; $\mu$ is Poisson's ratio, $\kappa=(0.87+$ $1.12 \mu)^{2} /(1+\mu)^{2}$ is the shear correction factor, and $G=E /(2+$ $2 \mu$ ) is the shear modulus. $A, \rho$, and $I$ are the cross-sectional area, density, and inertia moment of the beam, respectively.

By solving (1)-(4), the spectral solutions of the Timoshenko beam can be expressed as

$$
\begin{aligned}
u(x, \omega)= & A_{1} e^{-i k_{3} x}+A_{2} e^{-k_{3}\left(L_{b}-x\right)}, \\
w(x, \omega)= & A_{3} e^{-i k_{1} x}+A_{4} e^{-k_{1}\left(L_{b}-x\right)} \\
& +A_{5} e^{-i k_{2} x}+A_{6} e^{-k_{2}\left(L_{b}-x\right)}, \\
\varphi(x, \omega)= & \alpha_{1} A_{3} e^{-i k_{1} x}-\alpha_{1} A_{4} e^{-k_{1}\left(L_{b}-x\right)} \\
& +\alpha_{2} A_{5} e^{-i k_{2} x}-\alpha_{2} A_{6} e^{-k_{2}\left(L_{b}-x\right)},
\end{aligned}
$$

where the length of the beam is $L_{b}, A_{i}(i=1, \ldots, 6)$ is the spectral amplitude, $\omega$ is the frequency, $\alpha_{j}=\left(k_{j}^{2} \kappa G-\right.$ $\left.\rho \omega^{2}\right) /\left(i k_{j} \kappa G\right), j=1,2$, and $k_{1}, k_{2}$, and $k_{3}$ are wave numbers of bending mode and stretching mode, respectively, which can be expressed as

$$
k_{1,2}=\frac{1}{\sqrt{2}} \sqrt{x_{1} \pm \sqrt{x_{1}^{2}-4 x_{2}}}, \quad k_{3}=\omega \sqrt{\frac{\rho}{E}},
$$

where $x_{1}=\rho \omega^{2}[1 /(\kappa G)+1 / E]$ and $x_{2}=\rho \omega^{4} /(E \kappa G)-$ $\rho A \omega^{2} /(E I)$.

The coefficients $A_{i}(i=1, \ldots, 6)$ can be calculated as a function of the nodal spectral displacements using the boundary conditions. The nodal spectral forces can be determined by differentiating the spectral displacements with respect to $x$. Finally, using relation between the nodal forces and nodal displacements the spectral element stiffness matrix of an intact Timoshenko beam can be obtained as shown in [18]. 


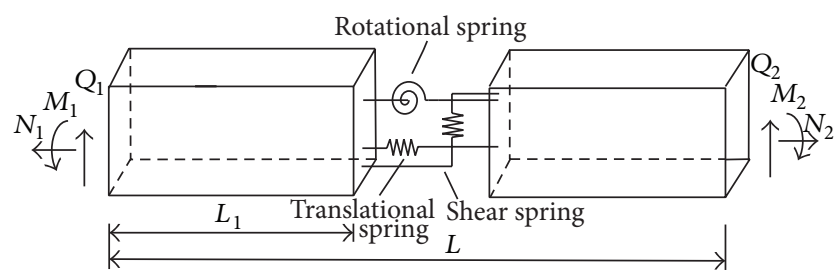

FIGURE 1: Spectral beam element model of cracked beam segment.

\subsection{Spectral Element Stiffness Matrix of a Cracked Timoshenko} Beam. The spectral element stiffness matrices of beam segments with and without a crack are similar in form. A spectral beam element model with a transverse open and nonpropagating crack is presented as shown in Figure 1. The crack is simulated by three massless springs, namely, one rotational spring, one translational spring, and one shear spring in this paper, whose flexibilities are calculated using Castigliano's theorem and the laws of the fracture mechanics. The length of the beam element is $L$ and the crack is located at $L_{1}$.

The spectral solutions for the left and right parts of the beam divided by the crack are similar to the form of (5), which can be expressed using a local coordinate system as

$$
\begin{aligned}
& u_{1}(x, \omega)= A_{1} e^{-i k_{3} x}+A_{2} e^{-k_{3}\left(L_{1}-x\right)}, \\
& \begin{aligned}
& w_{1}(x, \omega)= A_{3} e^{-i k_{1} x}+A_{4} e^{-k_{1}\left(L_{1}-x\right)}+A_{5} e^{-i k_{2} x} \\
&+A_{6} e^{-k_{2}\left(L_{1}-x\right)}, \\
& \varphi_{1}(x, \omega)= \alpha_{1} A_{3} e^{-i k_{1} x}-\alpha_{1} A_{4} e^{-k_{1}\left(L_{1}-x\right)} \\
&+\alpha_{2} A_{5} e^{-i k_{2} x}-\alpha_{2} A_{6} e^{-k_{2}\left(L_{1}-x\right)}, \\
& x \in\left(0, L_{1}\right), \\
& u_{2}(x, \omega)= A_{7} e^{-i k_{3}\left(L_{1}+x\right)}+A_{8} e^{-k_{3}\left(L-L_{1}-x\right)}, \\
& w_{2}(x, \omega)= A_{9} e^{-i k_{1}\left(L_{1}+x\right)}+A_{10} e^{-k_{1}\left(L-L_{1}-x\right)} \\
&+A_{11} e^{-i k_{2}\left(L_{1}+x\right)}+A_{12} e^{-k_{2}\left(L-L_{1}-x\right)}, \\
& \varphi_{2}(x, \omega)= \alpha_{1} A_{9} e^{-i k_{1}\left(L_{1}+x\right)}-\alpha_{1} A_{10} e^{-k_{1}\left(L-L_{1}-x\right)} \\
&+\alpha_{2} A_{11} e^{-i k_{2}\left(L_{1}+x\right)}-\alpha_{2} A_{12} e^{-k_{2}\left(L-L_{1}-x\right)}, \\
& x \in\left(0, L-L_{1}\right) .
\end{aligned}
\end{aligned}
$$

Node displacements at the two ends of the spectral cracked beam element are

$$
\begin{array}{lll}
\left.u_{1}\right|_{x=0}=u_{e 1}, & \left.w_{1}\right|_{x=0}=w_{e 1}, & \left.\varphi_{1}\right|_{x=0}=\varphi_{e 1}, \\
\left.u_{2}\right|_{x=L}=u_{e 2}, & \left.w_{2}\right|_{x=L}=w_{e 2}, & \left.\varphi_{2}\right|_{x=L}=\varphi_{e 2} .
\end{array}
$$

The continuity conditions at the crack location are

$$
\begin{gathered}
\left.\frac{\partial u_{1}}{\partial x}\right|_{x=L_{1}}=\left.\frac{\partial u_{2}}{\partial x}\right|_{x=0}, \\
\left.E A \frac{\partial u_{1}}{\partial x}\right|_{x=L_{1}}=k_{11}\left(\left.u_{2}\right|_{x=0}-\left.u_{1}\right|_{x=L_{1}}\right) \\
+k_{13}\left(\left.\varphi_{2}\right|_{x=0}-\left.\varphi_{1}\right|_{x=L_{1}}\right), \\
\kappa G A\left(\left.\frac{\partial w_{1}}{\partial x}\right|_{x=L_{1}}-\left.\varphi_{1}\right|_{x=L_{1}}\right) \\
=k_{22}\left(\left.w_{2}\right|_{x=0}-\left.w_{1}\right|_{x=L_{1}}\right), \\
=k_{31}\left(\left.u_{2}\right|_{x=0}-\left.u_{1}\right|_{x=L_{1}}\right) \\
+k_{33}\left(\left.\varphi_{2}\right|_{x=0}-\left.\varphi_{1}\right|_{x=L_{1}}\right), \\
\left.\frac{\left.\partial w_{1}\right|_{x=L_{1}}}{-} \varphi_{1}\right|_{x=L_{1}}=\left.\frac{\partial w_{2}}{\partial x}\right|_{x=0}-\left.\varphi_{2}\right|_{x=0}, \\
\left.\frac{\partial \varphi_{1}}{\partial x}\right|_{x=L_{1}}=\left.\frac{\partial \varphi_{2}}{\partial x}\right|_{x=0},
\end{gathered}
$$

where $k_{11}, k_{13}, k_{22}$, and $k_{33}$ are stiffness coefficientsof the three springs, respectively. Their values can be obtained by Castigliano's theorem [33]:

$$
\mathbf{k}=\left[\begin{array}{ccc}
k_{11} & 0 & k_{13} \\
0 & k_{22} & 0 \\
k_{13} & 0 & k_{33}
\end{array}\right]=\left[\begin{array}{ccc}
c_{11} & 0 & c_{13} \\
0 & c_{22} & 0 \\
c_{13} & 0 & c_{33}
\end{array}\right]^{-1},
$$

where $c_{11}, c_{13}, c_{31}$, and $c_{33}$ are the flexibility coefficients of the three springs, respectively, which can be expressed as

$$
\begin{aligned}
& c_{11}=\frac{2 \pi}{E b} \int_{0}^{\bar{a}} \bar{\alpha} F_{1}^{2}(\bar{\alpha}) d \bar{\alpha}, \\
& c_{22}=\frac{2 \pi \beta}{E b} \int_{0}^{\bar{a}} \bar{\alpha} F_{I I}^{2}(\bar{\alpha}) d \bar{\alpha}, \\
& c_{13}=\frac{12 \pi}{E b h} \int_{0}^{\bar{a}} \bar{\alpha} F_{1}(\bar{\alpha}) F_{2}(\bar{\alpha}) d \bar{\alpha}, \\
& c_{33}=\frac{72 \pi}{E b h^{2}} \int_{0}^{\bar{a}} \bar{\alpha} F_{2}^{2}(\bar{\alpha}) d \bar{\alpha},
\end{aligned}
$$

where $b$ is the width of the beam and $\bar{a}=h_{c} / h$ is the ratio between the crack depth $h_{c}$ and the height of the beam $h$. 


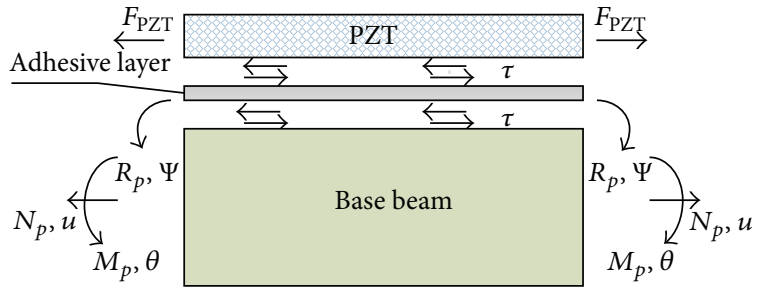

FIGURE 2: Smart beam element with surface-bonded PZT.

$\beta=10(1+\mu) /(12+11 \mu)$ denotes the shearing factor of the Timoshenko beam, and

$$
\begin{aligned}
& F_{1}\left(\frac{\alpha}{h}\right)=\frac{0.752+2.02(\alpha / h)+0.37[1-\sin (\pi \alpha / 2 h)]^{3}}{\cos (\pi \alpha / 2 h)}, \\
& F_{2}\left(\frac{\alpha}{h}\right)=\sqrt{\frac{2 h}{\pi \alpha} \tan \frac{\pi \alpha}{2 h} \frac{0.923+0.199[1-\sin (\pi \alpha / 2 h)]^{4}}{\cos (\pi \alpha / 2 h)},} \\
& F_{I I}\left(\frac{\alpha}{h}\right)=\frac{1.30-0.65(\alpha / h)+0.37(\alpha / h)^{2}+0.28(\alpha / h)^{3}}{\sqrt{1-\alpha / h}} .
\end{aligned}
$$

In addition, the boundary conditions of the free-free beam are expressed as $N_{1}^{(1)}=Q_{1}^{(1)}=M_{1}^{(1)}=N_{2}^{(n)}=Q_{2}^{(n)}=$ $M_{2}^{(n)}=0$.

After the above analysis, the stiffness matrix of the crack can be obtained. By substituting (7) into (8)-(9), the spectral nodal displacements can be written in a matrix form as follows:

$$
\mathbf{q}=\mathbf{D A}
$$

where $\mathbf{q}=\left[\begin{array}{llllllllllll}u_{e 1} & w_{e 1} & \varphi_{e 1} & 0 & 0 & 0 & 0 & 0 & 0 & u_{e 2} & w_{e 2} & \varphi_{e 2}\end{array}\right]^{T}$, $\mathbf{D}$ is provided in Appendix $\mathrm{A}$, and $\mathbf{A}=$ $\left[\begin{array}{llllllllllll}A_{1} & A_{2} & A_{3} & A_{4} & A_{5} & A_{6} & A_{7} & A_{8} & A_{9} & A_{10} & A_{11} & A_{12}\end{array}\right]^{T}$.

By substituting (7) into (4), the spectral nodal forces can be expressed in the matrix form as follows:

$$
\mathbf{F}=\mathbf{B A} \text {, }
$$

where $\mathbf{F}=\left[\begin{array}{llllll}N_{1} & Q_{1} & M_{1} & N_{2} & Q_{2} & M_{2}\end{array}\right]$ and $\mathbf{B}$ is provided in Appendix B.

The spectral force-displacement relation can be derived by eliminating the coefficient matrix $\mathbf{A}$ in (13) and (14) as follows:

$$
\mathbf{F}=\mathbf{B D}^{-1} \mathbf{q}=\mathbf{K q} \text {. }
$$

$\mathbf{K}$ is the frequency-dependent spectral element stiffness matrix of the Timoshenko beam with a crack. Crossing out 4 to 9 columns, the spectral stiffness matrixcan be written as a square matrix $(6 \times 6)$.

2.3. Spectral Element Stiffness Matrix of Beam Segment with Surface-Bonded PZT. The vibration properties of beam segment with surface-bonded PZT, which is a PZT-adhesive layer-beam coupled structural element, as shown in Figure 2, must be considered synthetically. Below are the equivalent parameters of the coupled structural element:

$$
\begin{gathered}
A_{e}=b h+b_{a} h_{a}+b_{p} h_{p}, \\
\rho_{e} A_{e}=\rho b h+\rho_{a} b_{a} h_{a}+\rho_{p} b_{p} h_{p}, \\
\rho_{e} I_{e}=\frac{\rho b h^{3}}{12}+\frac{\rho_{a} b_{a}}{12}\left[\left(h_{a}+h\right)^{3}-h^{3}\right]+\frac{\rho_{p} b_{p}}{12} \\
\times\left[\left(h_{p}+h_{a}+h\right)^{3}-\left(h+h_{a}\right)^{3}\right], \\
E_{e} I_{e}=\frac{E b h^{3}}{12}+\frac{E_{a} b_{a}}{12}\left[\left(h_{a}+h\right)^{3}-h^{3}\right]+\frac{Y_{11} b_{p}}{12} \\
\times\left[\left(h_{p}+h_{a}+h\right)^{3}-\left(h+h_{a}\right)^{3}\right],
\end{gathered}
$$

where $A_{e}, \rho_{e}, E_{e}$, and $I_{e}$ denote the equivalent cross-sectional area, density, elasticity modulus, and inertia moment of the coupled structure, respectively; $b, h, \rho, E$, and $I$ are the width, height, density, elasticity modulus, and inertia moment of the base beam, respectively; $b_{a}, h_{a}, \rho_{a}, E_{a}$, and $I_{a}$ are the width, height, density, elasticity modulus, and inertia moment of the adhesive layer, respectively; $b_{p}, h_{p}, \rho_{p}, Y_{11}$, and $I_{p}$ are the width, height, density, elasticity modulus, and inertia moment of the PZT patch, respectively.

Substituting these equivalent parameters into (7), the displacement equations of the beam segment with surfacebonded PZT can be obtained. According to the analysis method of cracked beam segment, the spectral element stiffness matrix of the beam segment with surface-bonded PZT can be also derived, whose form is similar to the cracked beam.

As shown in Figure 2, a single PZT is bonded onto the surface of the base beam. The piezoelectric smart beam consists of three layers: piezoelectric layer, adhesive layer, and base beam. The PZT will produce longitudinal expansion and shrink under voltage excitation. There exist interaction forces between the three layers. The base beam will produce both axial and bending vibration when there is only a single PZT. $N_{p}, M_{p}$, and $R_{p}$ denote the forces and moments applied to the base beam, which are generated by the driving voltage. $u, \theta$, and $\psi$ are the corresponding displacements. These forces and moments can be expressed in the spectral form as follows:

$$
\begin{gathered}
N_{p}=b_{p} d_{31} Y_{11} V(\omega), \\
M_{p}=b_{p} d_{31} Y_{11}\left(\frac{h}{2}+h_{a}+\frac{h_{p}}{2}\right) V(\omega), \\
R_{p}=b_{p} d_{31} Y_{11} h_{a} V(\omega) .
\end{gathered}
$$

As shown in Figures 3 and 4, two PZT patches are attached to the top and bottom surfaces of the beam 


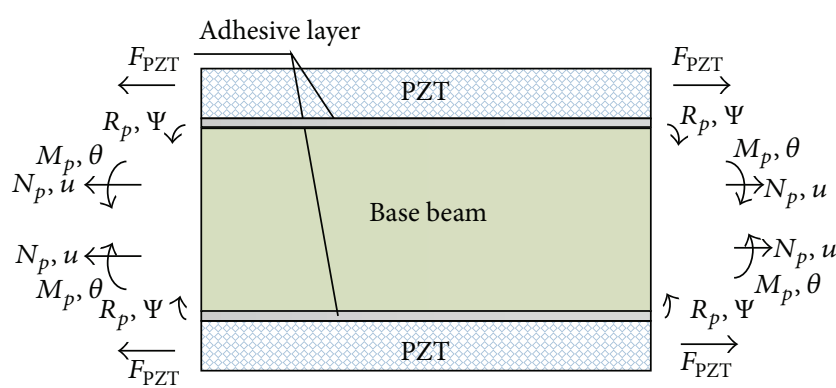

FIGURE 3: Smart beam element with collocated PZT patches under the same phase of electrical excitation.

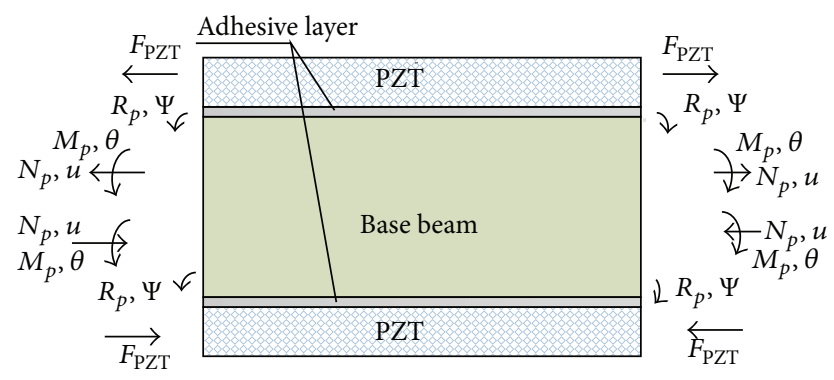

FIGURE 4: Smart beam element with collocated PZT patches under the opposite phase of electrical excitation.

symmetrically. The vibration in this case is different to the case that only a single PZT is boned onto the top surface of the base beam.

Under the same size and same phase of the electrical excitation, bending vibration caused by the two PZT patches will be offset; the base beam only produces axial vibration. In this case, forces and moments applied to the base beam are only $2 N_{p}$. Details of the forces can be seen in Figure 3.

On the contrary, when the PZT patches are under the same size but opposite phase of the electrical excitation, axial vibration caused by the two PZT patches will be offset; the base beam only produces bending vibration. In this case, forces and moments applied to the base beam are $2 M_{p}$ and $2 R_{p}$, which can be concluded from Figure 4 clearly.

2.4. Formation of Global Stiffness Matrix. The PZT-adhesive layer-cracked beam coupled structure can be divided into different segments due to the location of PZT patch. The spectral element stiffness matrices of beam segments with and without a crack are similar in form. The spectral element stiffness matrix of beam segments with PZT is also derived. So the stiffness matrix of the beam can be determined.

Continuity conditions between different beam segments can be expressed as

$$
\begin{array}{ccc}
N_{2}^{(i)}=N_{1}^{(i+1)}, & Q_{2}^{(i)}=Q_{1}^{(i+1)}, & M_{2}^{(i)}=M_{1}^{(i+1)}, \\
u_{e 2}^{(i)}=u_{e 1}^{(i+1)}, & w_{e 2}^{(i)}=w_{e 1}^{i+1}, & \varphi_{e 2}^{(i)}=\varphi_{e 1}^{(i+1)},
\end{array}
$$

where superscript " $i$ " denotes the number of the beam segments, subscript " 1 " denotes the left end of the beam segment, and subscript " 2 " denotes the right end of the beam segment. By assembling stiffness matrices of different beam segments through the above continuity conditions, the global spectral element stiffness matrix of the piezoelectric smart beam can be obtained.

In order to analyze the displacement of the piezoelectric smart beam under load, restraint conditions at the two ends need to be considered. When one end of the beam is free, to the left end as an example, there exists $N_{1}=Q_{1}=M_{1}=0$; when it is simply supported, then $M_{1}=u_{e 1}=w_{e 1}=0$; when it is fixed, then $u_{e 1}=w_{e 1}=\varphi_{e 1}=0$. By taking a different combination of the above restraints, different boundary conditions of the piezoelectric smart beam can be simulated.

\section{EMI Analysis on Piezoelectric Smart Beam}

A single PZT patch is bonded onto the top surface of the piezoelectric beam. Relative to the base beam, the size of piezoelectric patch is very small. The PZT is considered as a one-dimensional model and the force generated by piezoelectric patches can be simplified as a pair of axial force. The constitutive equations of the PZT patch are

$$
\begin{gathered}
\varepsilon_{1}=\frac{\sigma_{1}}{\bar{Y}_{11}}+d_{31} E_{3}, \\
D_{3}=\bar{\varepsilon}_{33} E_{3}+d_{31} \sigma_{1},
\end{gathered}
$$

where $\varepsilon_{1}, \sigma_{1}$ are the strain and stress, respectively; $\bar{Y}_{11}=$ $Y_{11}(1+\eta j)$ is complex Young's modulus at zero electric field with $\eta$ denoting the mechanical loss factor; $E_{3}=V(\omega) / h_{p}$ is the electric field intensity; $D_{3}$ is the electric displacement along $z$ direction, respectively; $\bar{\varepsilon}_{33}=\varepsilon_{33}(1-\delta j)$ is the complex dielectric constant at zero stress with $\eta$ denoting the dielectric loss factor; $d_{31}$ is the piezoelectric strain constant.

From (19), the electric displacement and the electric displacement can be defined as

$$
D_{3}=d_{31} \bar{Y}_{11} \varepsilon_{1}+\left(\bar{\varepsilon}_{33}-d_{31}^{2} \bar{Y}_{11}\right) E_{3}
$$

The electric current passing through the PZT patch can be obtained from the electric displacement as follows:

$$
\begin{aligned}
I(\omega)= & i \omega \iint D_{3} d A \\
= & i \omega d_{31} Y_{11} b_{p}\left[\bar{U}-\left(\frac{h}{2}+h_{a}+\frac{h_{p}}{2}\right) \bar{\theta}+h_{a} \bar{\psi}\right] \\
& +i \omega b_{p} l_{p}\left(\varepsilon_{33}-d_{31}^{2} Y_{11}\right) \frac{V(\omega)}{h_{p}},
\end{aligned}
$$




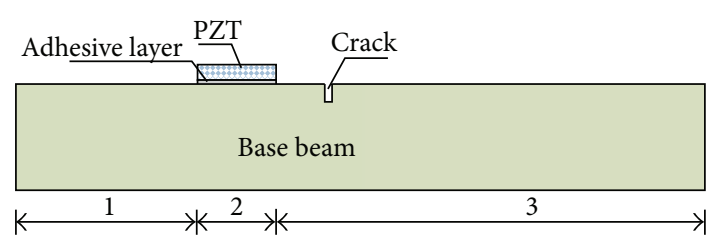

FIGURE 5: Piezoelectric smart beam with a crack and a single PZT patch.

where $l_{p}$ denotes the length of the PZT patch; $\bar{U}, \bar{\theta}$, and $\bar{\psi}$ are the change of axial displacement, rotational angle, and shear displacement of two ends of the bonded-PZT beam, which can be expressed as

$$
\begin{gathered}
\bar{U}=\left.u\right|_{x=L_{p}}-\left.u\right|_{x=0}, \quad \bar{\theta}=\left.\theta\right|_{x=L_{p}}-\left.\theta\right|_{x=0}, \\
\bar{\psi}=\left.\psi\right|_{x=L_{p}}-\left.\psi\right|_{x=0} .
\end{gathered}
$$

The coupled EMI of the piezoelectric smart beam can be described as

$$
Z(\omega)=\frac{V(\omega)}{I(\omega)}
$$

For the piezoelectric smart beam, the PZT measured impedance curve is the function of the dynamic characteristic of PZT patch and impedance value of the base beam. It can be found that the impedance of the piezoelectric smart beam has relation with parameters of the beam. When the beam is damaged, the impedance curve of the piezoelectric smart beam will change. So change of the electromechanical impedance of the beam can reflect damage of the beam.

\section{Experimental Verification}

In this study, a free-free Timoshenko beam with a single PZT patch attached to its top surface is investigated. The type of PZT patch is PZT5A, and the PZT is located at $x_{1}=$ $136.5 \mathrm{~mm}, x_{2}=163 \mathrm{~mm}$ from the left end of the beam, and the crack is located at $x_{3}=182 \mathrm{~mm}$. Here we consider intact beam and three damage cases, which are corresponding to the relative crack depths of $10 \%, 20 \%$, and $30 \%$, respectively. The detailed information is as shown in Figure 5; a single PZT patch is bonded onto the top surfaces of a Timoshenko beam with a crack. The PZT-adhesive layer-cracked beam coupled structure is divided into three segments due to the location of PZT patch. The material constants of the beam, adhesive layer, and PZT patch are listed in Table 1.

The PZT will produce longitudinal expansion and shrink under 1 voltage excitation, and then the base beam will also produce vibration. The realimpedances of intact smart beam are calculated using SEM in two different frequency ranges of $10-30 \mathrm{kHz}$ and $80-100 \mathrm{kHz}$. In order to verify the validity of the analysis results using SEM, some experimental results for intact smart beam are also used for comparison. Numerical and experimental real impedance curves of smart intact beam and cracked beam are shown in Figures 6 and 7, respectively.

As shown in Figures 6 and 7, numerical and experimental results are in agreement by and large, and the impedance magnitudes are within an order of magnitude. Parts of the wave locations do not match. There are many reasons for this case. There exists error usingfoam to simulate free boundary conditions. Numerical models of piezoelectric patch and adhesive layer are considered too simple, which cannot fully reflect the actual vibration conditions. From the analysis results, it is found that the peak position and amplitude of the electromechanical impedance curve have significant changes with the crack, especially in higher frequency ranges. The experimental results are coincident with those using SEM. Therefore, it can be concluded that the SEM can be used to analyze the electromechanical impedance responses of a piezoelectric smart beam effectively.

\section{Numerical Analysis}

5.1. Different Damage Cases. The crack is simulated by three massless springs, namely, one rotational spring, one translational spring, and one shear spring in this paper. In order to certify the effectiveness of the spring model, piezoelectric beam under different damage conditions is analyzed. Because the process of vibration with a single PZT is too complicated, and the number of axial vibration modes is relatively small, therefore, the bending mode is taken to analyze the effect of crack depth on the admittance of the beam in this paper. On the other hand, the peak of bending vibration mode is more significant than the other two modes, so it is more suitable to be applied in structural health monitoring.

The real impedances for piezoelectric smart beam with a crack are calculated using SEM in two different frequency ranges of $10-30 \mathrm{kHz}$ and $80-100 \mathrm{kHz}$. Numerical EMI signatures of smart beam with different crack sizes are shown in Figure 8. From Figure 8 it is found that the peak positions and amplitudes of the real impedance curves have significant left shifting trend with the variations of crack size, especially in higher frequency ranges. This is because the extension of cracks reduced the overall stiffness of the beam. That is, the greater the damage is, the more the curve shifts to the left. Therefore, better results can be achieved using high frequency for damage identification in the health monitoring.

5.2. Influence of Adhesive Layer. The impact of the adhesive layer has been considered when analyzing the impedance of the piezoelectric smart beam. When the thickness of the adhesive layer changes, the impedance of the piezoelectric beam will also change. In order to analyze the effect of thickness of adhesive layer on the impedance, free-free beam without crack is taken as the object. The cumulative admittance shifts (CAS) and the cross correlation coefficients 
TABLE 1: Material constants.

\begin{tabular}{lccccccccc}
\hline & $l_{b}(\mathrm{~mm})$ & $b_{b}(\mathrm{~mm})$ & $h_{b}(\mathrm{~mm})$ & $\rho_{b}\left(\mathrm{~kg} / \mathrm{m}^{3}\right)$ & $E\left(10^{9} \mathrm{~Pa}\right)$ & $\mu$ & $Y_{11}\left(10^{9} \mathrm{~Pa}\right)$ & $d_{31}\left(10^{-12} \mathrm{~m} / \mathrm{V}\right)$ & $\varepsilon_{33}\left(10^{-8} \mathrm{~F} / \mathrm{m}\right)$ \\
\hline PZT & 26.5 & 13.5 & 0.5 & 7860 & - & - & 60.16 & -143 & 1.311 \\
Adhesive & 26.5 & 13.5 & 0.02 & 1700 & 1 & - & - & - & - \\
Beam & 400 & 20 & 15 & 7800 & 210 & 0.3 & - & - \\
\hline
\end{tabular}

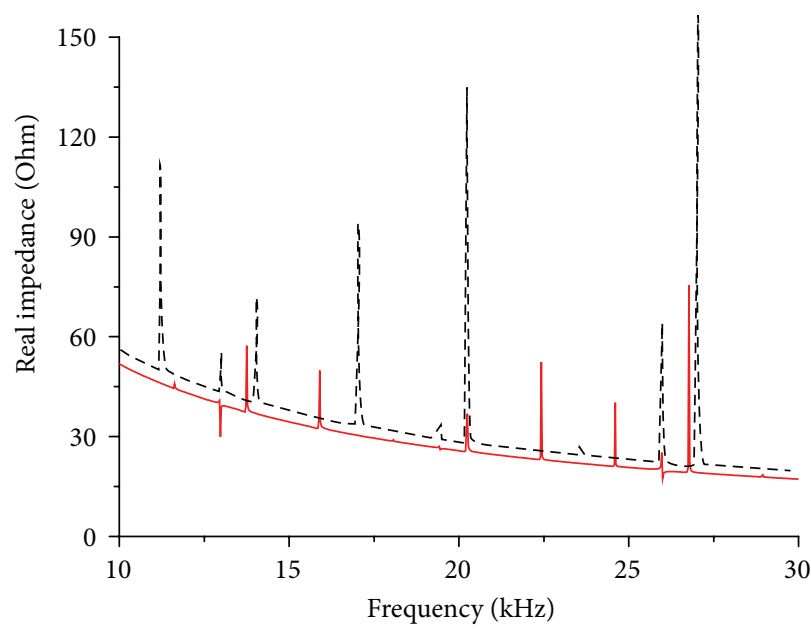

(a)

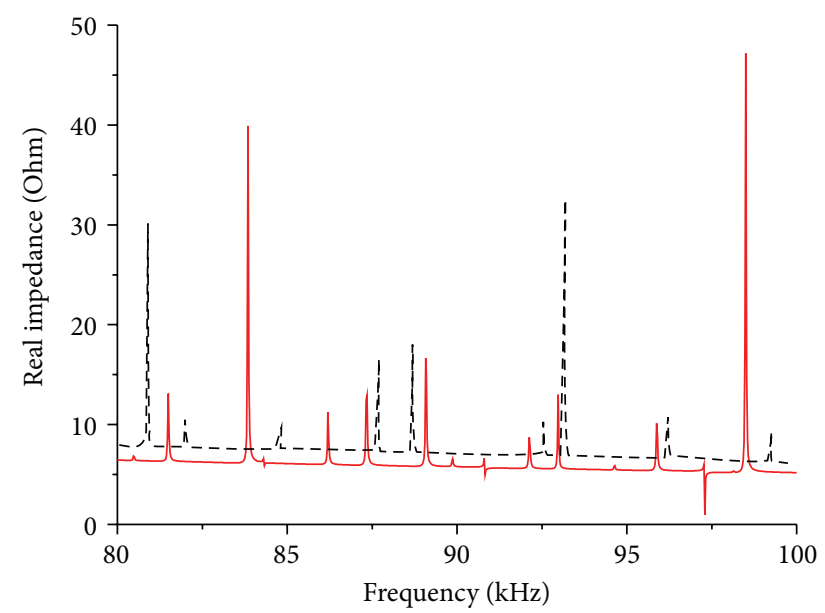

(b)

FigURE 6: EMI of the beam without a crack in different frequency ranges (- - -: experimental result, —: numerical result): (a) 10-30 kHz and (b) $80-100 \mathrm{kHz}$.

(CC) between two real admittance data are defined as

$$
\begin{gathered}
\mathrm{CAS}=\sum_{i=1}^{N} \frac{\operatorname{Re}\left(Y_{i}\right)-\operatorname{Re}\left(Y_{0}\right)}{\operatorname{Re}\left(Y_{0}\right)}, \\
\mathrm{CC}=\frac{1}{N-1} \sum_{i=1}^{N} \frac{\left[\operatorname{Re}\left(Z_{i}\right)-\overline{\operatorname{Re}\left(Z_{i}\right)}\right]\left[\operatorname{Re}\left(Z_{0}\right)-\overline{\operatorname{Re}\left(Z_{0}\right)}\right]}{\sigma_{\operatorname{Re}\left(Z_{i}\right)} \sigma_{\operatorname{Re}\left(Z_{0}\right)}}
\end{gathered}
$$

where $\operatorname{Re}\left(Y_{i}\right)$ and $\operatorname{Re}\left(Y_{0}\right)$ denote a real part of electric admittance with and without an adhesive layer, respectively; $N$ denotes the number of measurements; $\operatorname{Re}(Z)$ represents the real impedance; subscript "0" represents initial structural status without adhesive layer; superscript “__ represents the mean of a $\operatorname{Re}(Z) ; \sigma_{\operatorname{Re}\left(Z_{i}\right)}$ and $\sigma_{\operatorname{Re}\left(Z_{0}\right)}$ are the standard deviations of the real parts of $Z_{i}$ and $Z_{0}$.

As shown in Figure 9, with increasing thickness of the adhesive, the index CC is becoming smaller, and the change of correlation coefficients in the high frequency is more obvious than in the low frequency. When the adhesive layer thickness increases, force and moment applied to the beam become larger; the magnitude of the admittance will also increase. The cumulative admittance shifts (CAS) are used to analyze the change of magnitude of the admittance. The analysis results in two different frequency ranges of $10-30 \mathrm{kHz}$ and $80-100 \mathrm{kHz}$ can be seen in Figure 9(b).
5.3. Different Vibration Modes. When the PZT patches are pasted with different forms, the beam will produce different vibration modes. When pasted with a single PZT patch, the beam will produce both bending vibration and axial vibration. When two PZT patches attach to the top and bottom surfaces of the beam symmetrically, the vibration is different to the vibration of beam with a single PZT bonded onto it. Detail forces of the three vibration modes of the beam have been analyzed in the previous text. Under the same size and same phase of the electrical excitation, the base beam only produces axial vibration. Under the same size but opposite phase of the electrical excitation, the base beam only produces bending vibration. The three graphs in Figure 10 describe the vibration of the base beam with a single PZT bonded onto it, the vibration of the beam with collocated PZT patches under the same phase of electrical excitation, and that under the opposite phase of electrical excitation, respectively. The parameters of the beam are the same as those shown in Table 1 .

As shown in Figure 11, the number of modes causing vibration of the base beam with a single PZT is equal to the number of bending vibration plus the number of axial vibration with collocated $\mathrm{PZT}$ patches in frequency ranges of $10-30 \mathrm{kHz}$ and $80-100 \mathrm{kHz}$. This is because the beam will produce both bending vibration and axial vibration when only a single PZT patch vibrates under the voltage 


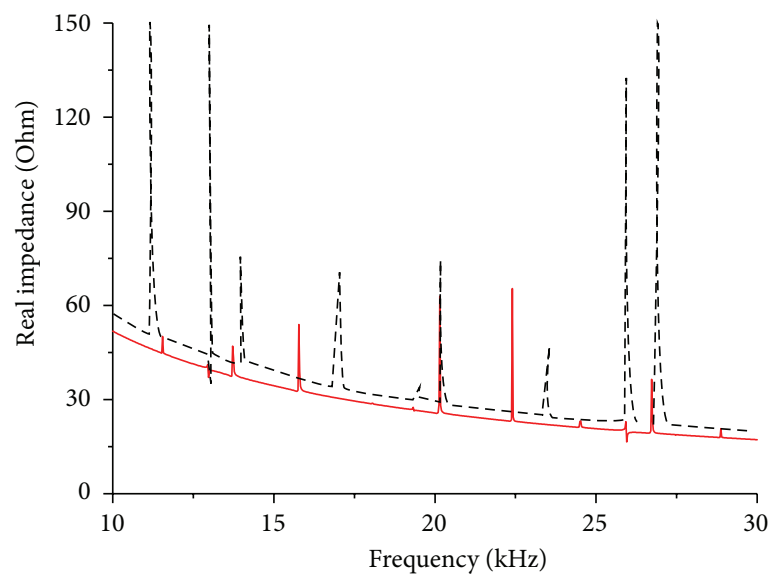

(a)

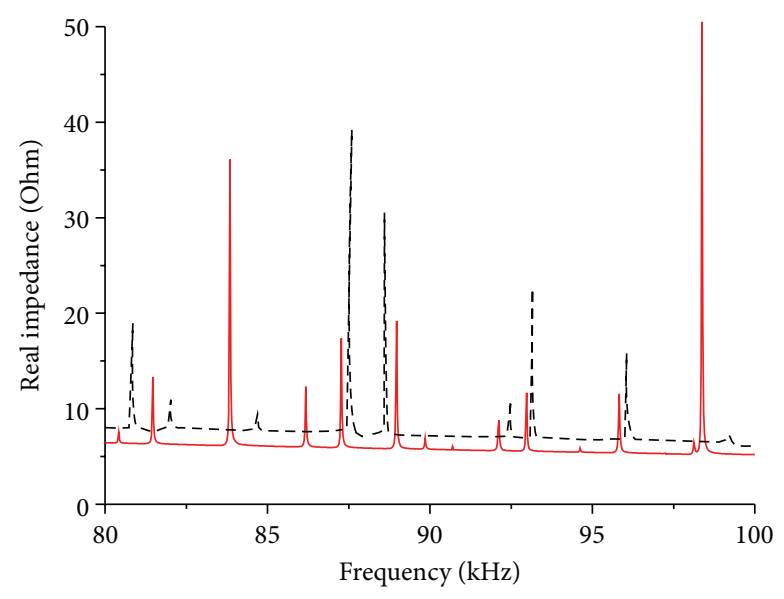

(b)

FIGURE 7: EMI of the beam with 10\% crack in different frequency ranges (- - -: experimental result, —: numerical result): (a) 10-30 kHz and (b) $80-100 \mathrm{kHz}$.

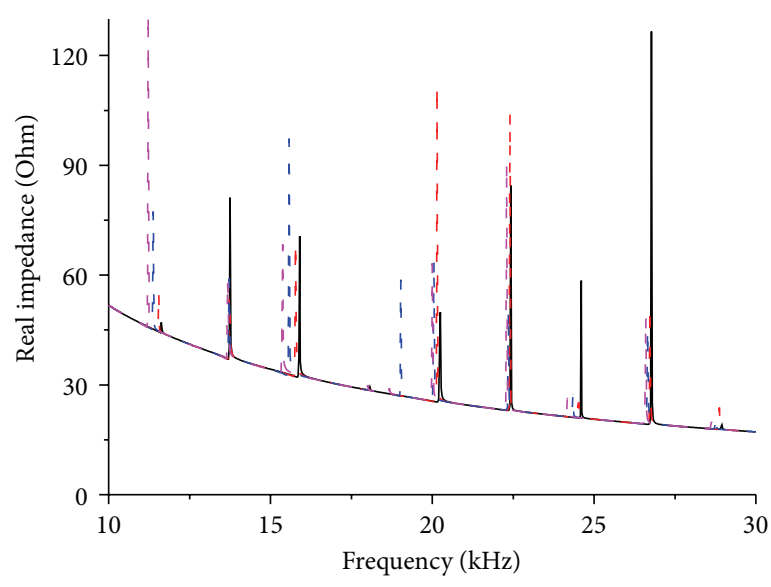

$-0$
$---20 \%$

$---30 \%$

(a)
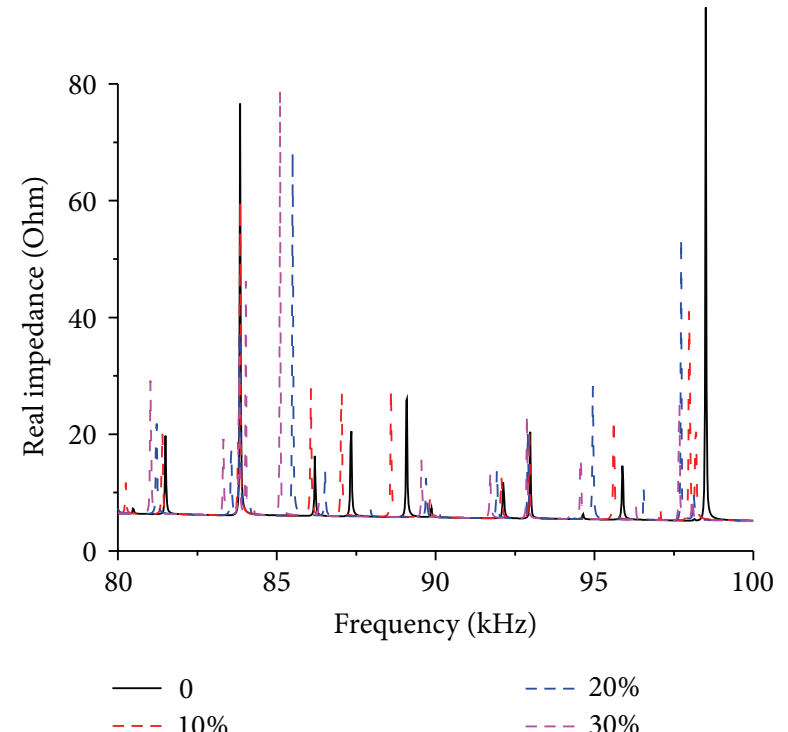

(b)

FIGURE 8: Real impedance of the smart beam with different crack sizes: (a) $10-30 \mathrm{kHz}$ and (b) $80-100 \mathrm{kHz}$.

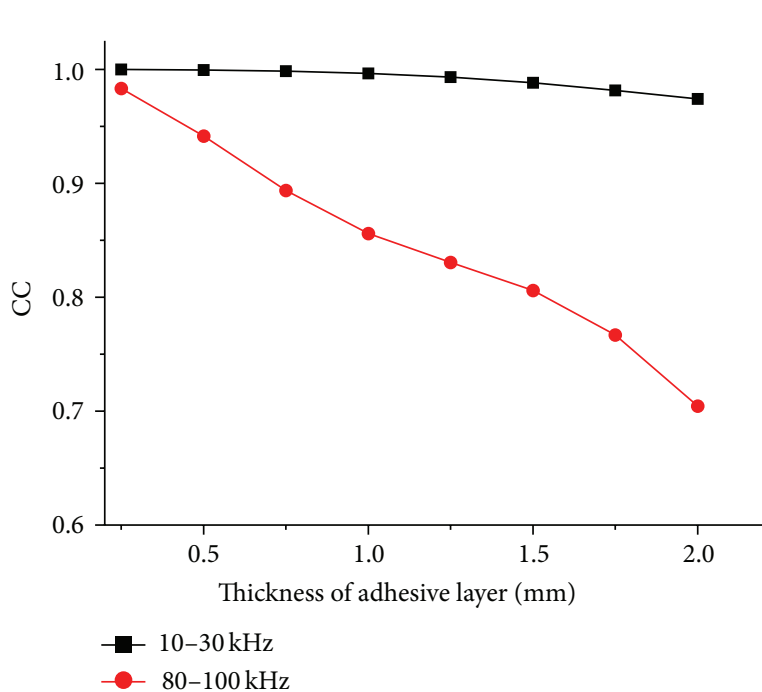

(a)

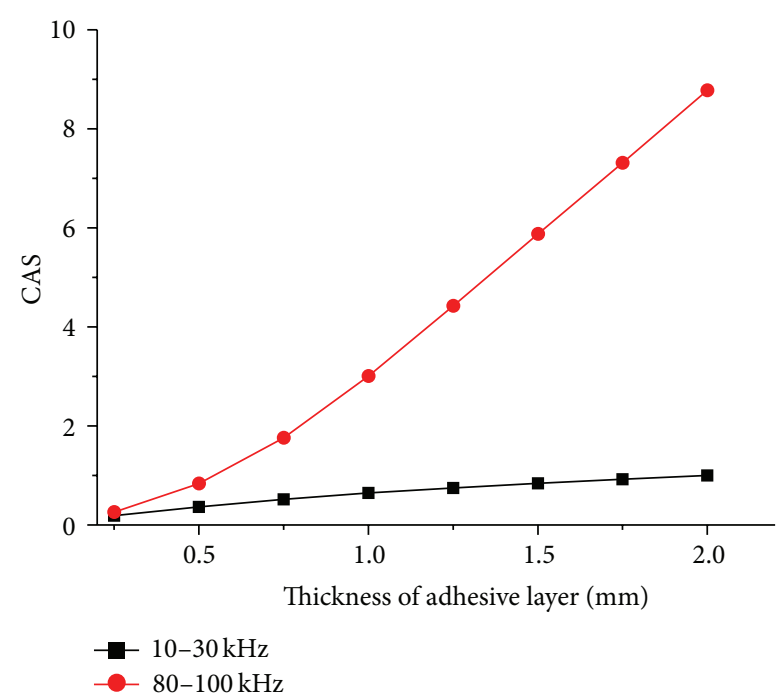

(b)

FIGURE 9: CC and CAS values of the piezoelectric smart beam in different frequency ranges: (a) CC index and (b) CAS index. 


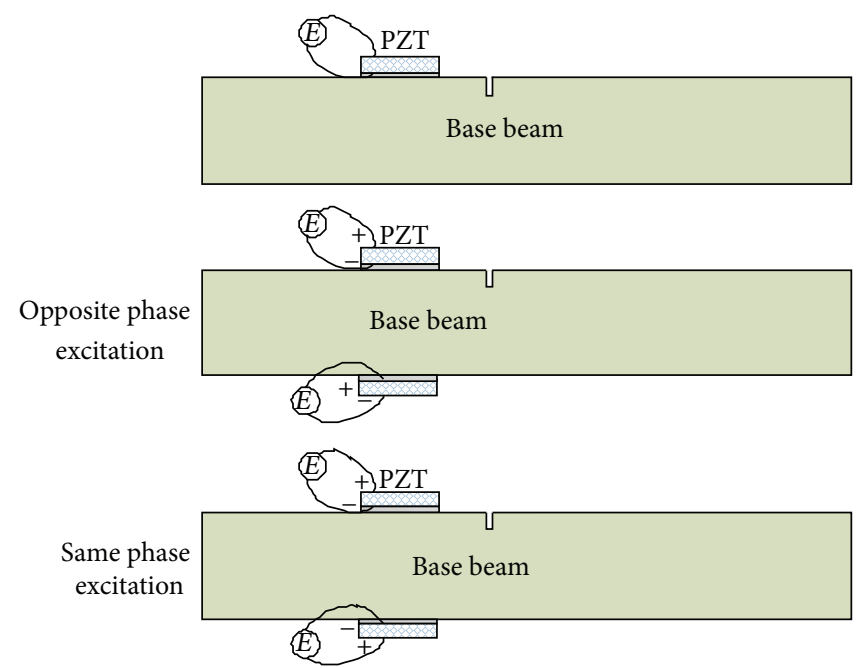

FIGURE 10: Piezoelectric smart beam with different PZT pasted forms.
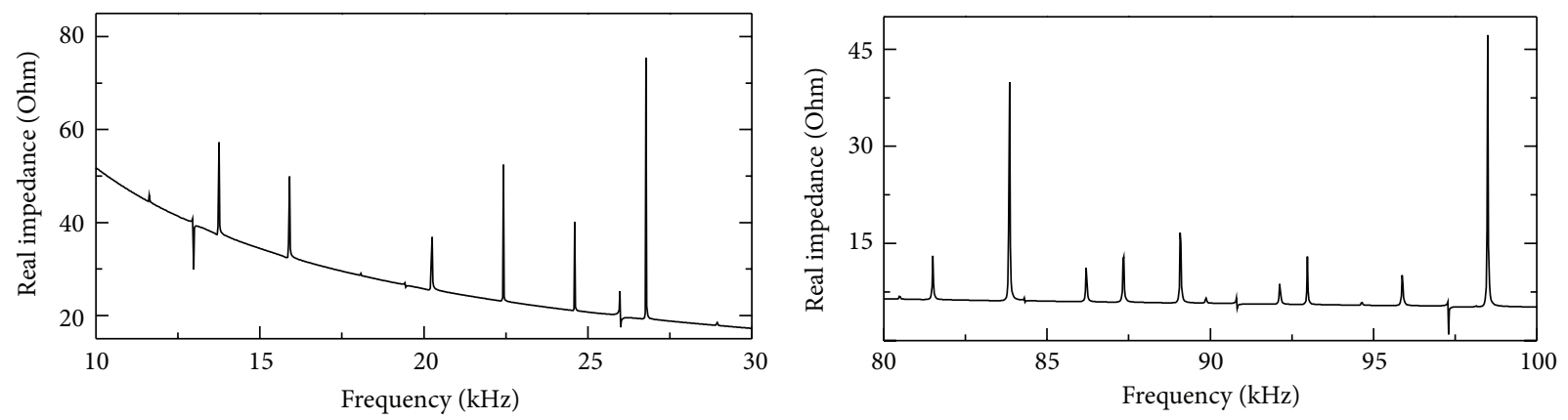

— Vibration with a single PZT
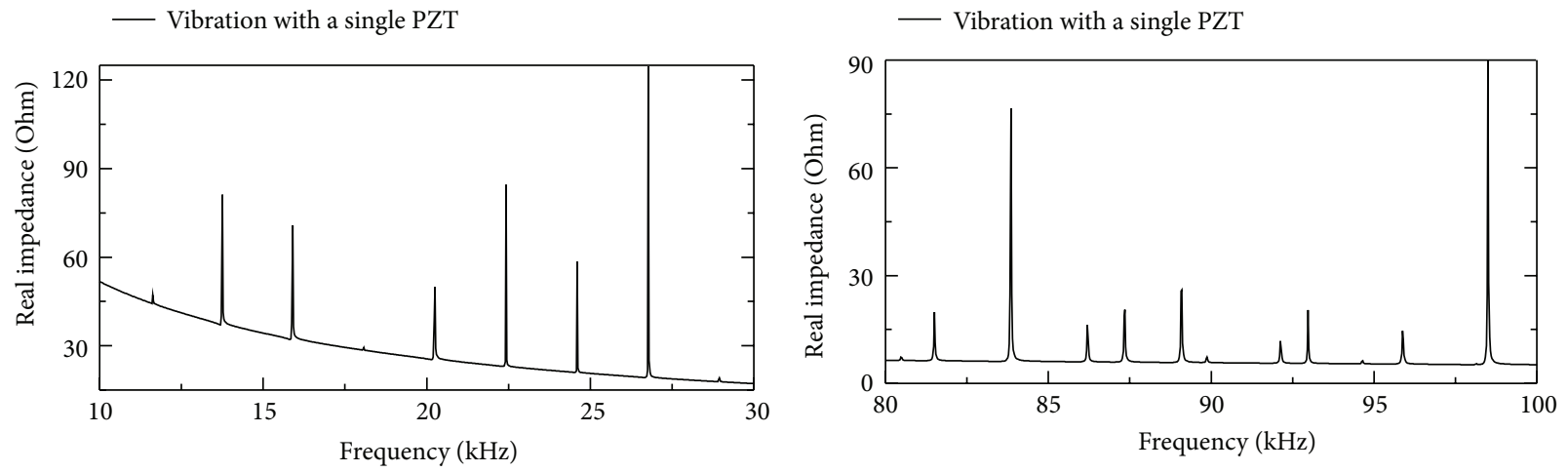

— Bending vibration
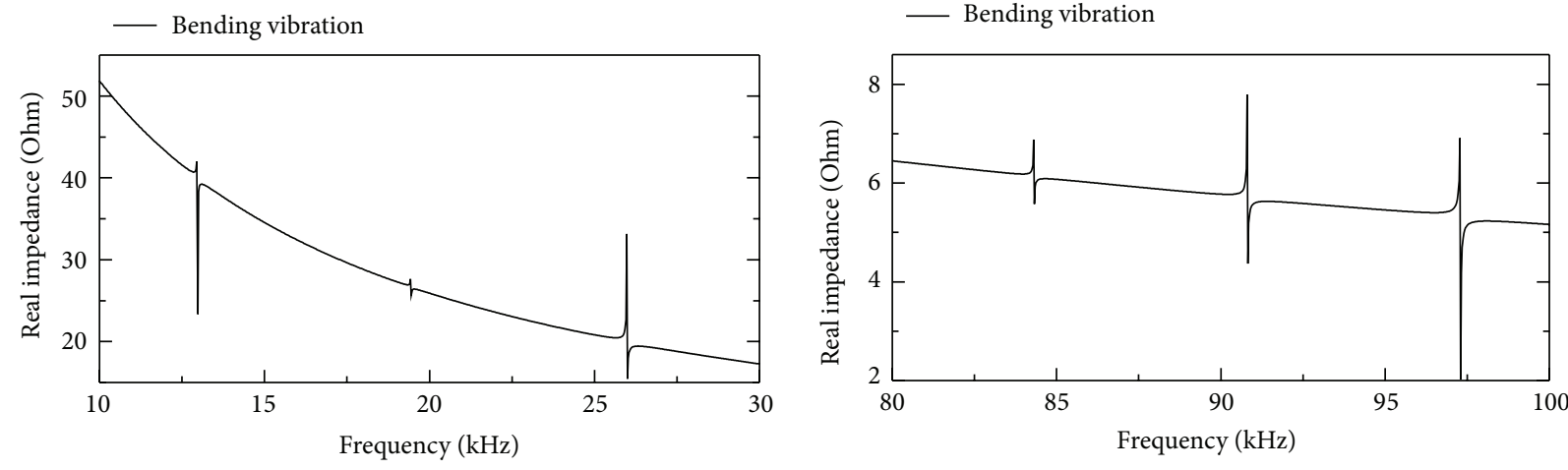

— Axial vibration

— Axial vibration

(a)

(b)

FIGURE 11: Real impedance of the free-free beam under different vibration modes: (a) $10-30 \mathrm{kHz}$ and (b) $80-100 \mathrm{kHz}$. 


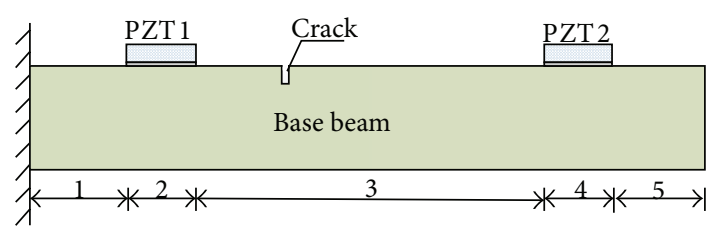

FIgURE 12: Piezoelectric smart beam with a crack and two PZT patches.

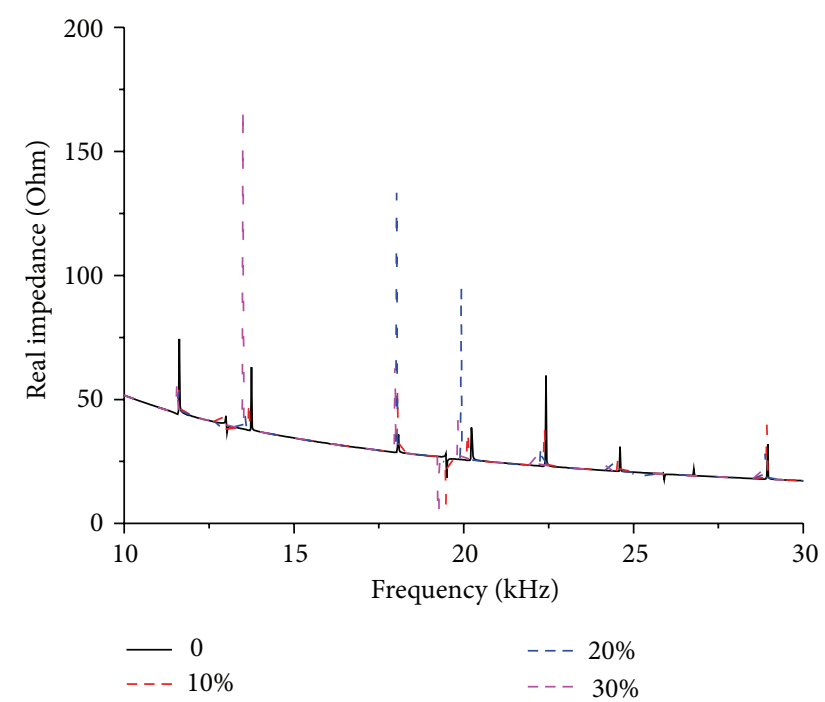

(a)

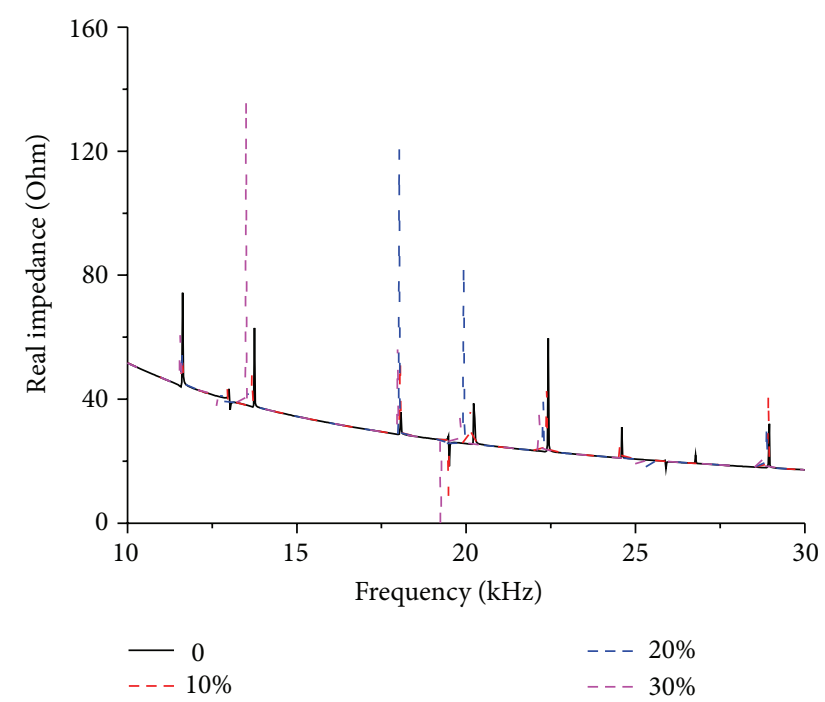

(b)

FIGURE 13: Real impedance of the smart beam with different crack sizes: (a) PZT1 and (b) PZT2.

excitation; the modes reflect information of bending vibration and axial vibration. On the other hand, the number of modes caused by the bending vibration is more than that caused by the axial vibration. This is because the value

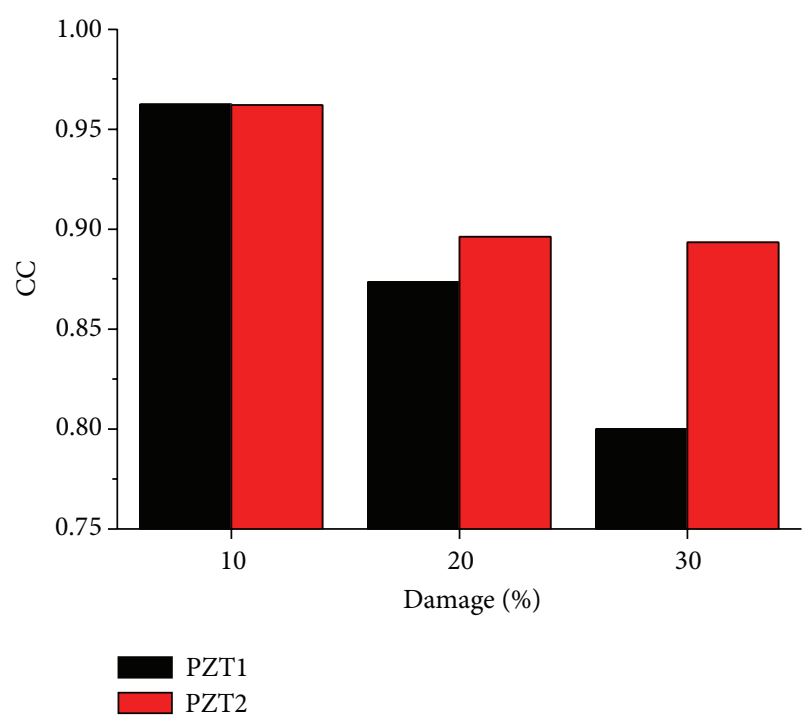

FIGURE 14: CC of two PZT patches with different crack sizes in the frequency range of $10-30 \mathrm{kHz}$.

of the area moment of inertia is much smaller than the sectional area, so in a certain frequency band the interval between bending modes is smaller than the axial vibration modes.

5.4. Smart Beam with Dual PZT Patches. In addition to the above analysis of a free-free beam, another beam with different damage conditions and patch pasted forms can be also analyzed. As shown in Figure 12, a cantilever Timoshenko beam with two PZT patches attached to its top surface is investigated. The PZT-adhesive layer-cracked beam coupled structure is divided into five segments due to the location of PZT patch. The length, width, and height of the piezoelectric smart beam are $400 \mathrm{~mm}, 20 \mathrm{~mm}$, and $15 \mathrm{~mm}$, respectively. PZT1 is located at $x_{1}=73.5 \mathrm{~mm}$ and $x_{2}=100 \mathrm{~mm}$ from the left end of the beam, PZT2 is located at $x_{1}=300 \mathrm{~mm}$ and $x_{2}=326.5 \mathrm{~mm}$ from the left end of the beam, and the crack is located at $x_{3}=150 \mathrm{~mm}$. Here we consider intact beam and three damage cases, which are corresponding to the relative crack depths of $10 \%, 20 \%$, and $30 \%$, respectively.

As shown in Figures 13 and 14, the real impedance curves of PZT1 and PZT2 have significant left shifting trend with the variations of crack size, and the left shifting trend of impedance curves of PZT1 is more significant than that of PZT2. That is because the position of PZT1 is closer to crack than PZT2, so the results are more sensitive to damage.

\section{Conclusions}

An impedance analysis of a piezoelectric smart beam with a crack and a single PZT patch attached to it is implemented in this paper. The crack is simulated by spring models, and the influence of adhesive layer is also considered. 
Different vibration modes of the piezoelectric beam are analyzed in this paper, and the effect of thickness of the adhesive layer on the admittance is also researched. In order to depict the effects of the depth of crack and thickness of adhesive layer on impedance curves of PZT patches, the damage index, cumulative admittance shifts (CAS), and cross correlation coefficient (CC) are defined. An experiment of a cracked beam with free boundary conditions at both ends is presented to verify the validity of the analysis results using SEM. The experimental and numerical results are in agreement by and large. It illustrates that using SEM to analyze the admittance of piezoelectric smart beam is feasible. Structural health monitoring technology based on electromechanical impedance using SEM has a certain application value.

\section{Appendices}

\section{A. Spectral Element Displacement Matrix}

Consider

D

$\left[\begin{array}{cccccc}1 & m & 0 & 0 & 0 & 0 \\ 0 & 0 & 1 & c & 1 & d \\ 0 & 0 & \alpha_{1} & -\alpha_{1} c & \alpha_{2} & -\alpha_{2} d \\ -i k_{3} m & i k_{3} & 0 & 0 & 0 & 0 \\ -k_{11} m+E A i k_{3} m & -k_{11}-E A i k_{3} & -k_{13} \alpha_{1} c & k_{13} \alpha_{1} & -k_{13} \alpha_{2} d & k_{13} \alpha_{2} \\ 0 & 0 & {\left[k_{22}+\xi\left(-i k_{1}-\alpha_{1}\right)\right] c} & k_{22}+\xi\left(i k_{1}+\alpha_{1}\right) & {\left[k_{22}+\xi\left(-i k_{2}-\alpha_{2}\right)\right] d} & k_{22}+\xi\left(i k_{2}+\alpha_{2}\right) \\ k_{13} m & k_{13} & \left(k_{33}-E I i k_{1}\right) \alpha_{1} c & \left(-k_{33}-E I i k_{1}\right) \alpha_{1} & \left(k_{33}-E I i k_{2}\right) \alpha_{2} d & \left(-k_{33}-E I i k_{2}\right) \alpha_{2} \\ 0 & 0 & \left(-i k_{1}-\alpha_{1}\right) c & i k_{1}+\alpha_{1} & \left(-i k_{2}-\alpha_{2}\right) d & i k_{2}+\alpha_{2} \\ 0 & 0 & i k_{1} \alpha_{1} c & i k_{1} \alpha_{1} & i k_{2} \alpha_{2} d & i k_{2} \alpha_{2} \\ 0 & 0 & 0 & 0 & 0 & 0 \\ 0 & 0 & 0 & 0 & 0 & 0\end{array}\right.$

$\left.\begin{array}{cccccc}0 & 0 & 0 & 0 & 0 & 0 \\ 0 & 0 & 0 & 0 & 0 & 0 \\ 0 & 0 & 0 & 0 & 0 & 0 \\ i k_{3} m & -i k_{3} n & 0 & 0 & 0 & 0 \\ k_{11} m & k_{11} n & k_{13} \alpha_{1} c & -k_{13} \alpha_{1} f & k_{13} \alpha_{2} d & -k_{13} \alpha_{2} g \\ 0 & 0 & -k_{22} c & -k_{22} f & -k_{22} d & -k_{22} g \\ -k_{13} m & -k_{13} n & -k_{33} \alpha_{1} c & k_{33} \alpha_{1} f & -k_{33} \alpha_{2} d & k_{33} \alpha_{2} g \\ 0 & 0 & \left(i k_{1}+\alpha_{1}\right) c & \left(-i k_{1}-\alpha_{1}\right) f & \left(i k_{2}+\alpha_{2}\right) d & \left(-i k_{2}-\alpha_{2}\right) g \\ 0 & 0 & -i k_{1} \alpha_{1} c & -i k_{1} \alpha_{1} f & -i k_{2} \alpha_{2} d & -i k_{2} \alpha_{2} g \\ p & 1 & 0 & 0 & 0 & 0 \\ 0 & 0 & a & 1 & b & 1 \\ 0 & 0 & \alpha_{1} a & -\alpha_{1} & \alpha_{2} b & -\alpha_{2}\end{array}\right], \quad a=e^{-i k_{2} L}$




\section{B. Spectral Element Force Matrix}

Consider

B

$$
=\left[\begin{array}{cccccccccccc}
E A i k_{3} & -E A i k_{3} m & 0 & 0 & 0 & 0 & 0 & 0 & 0 & 0 & 0 & 0 \\
0 & 0 & \frac{\rho A \omega^{2}}{i k_{1}} & -\frac{\rho A \omega^{2}}{i k_{1}} & \frac{\rho A \omega^{2}}{i k_{2}} & -\frac{\rho A \omega^{2}}{i k_{2}} & 0 & 0 & 0 & 0 & 0 & 0 \\
0 & 0 & E I k_{1} \alpha_{1} & E I i k_{1} \alpha_{1} c & E I i k_{2} \alpha_{2} & E I i k_{2} \alpha_{2} d & 0 & 0 & 0 & 0 & 0 & 0 \\
0 & 0 & 0 & 0 & 0 & 0 & -E A i k_{3} p & E A i k_{3} & 0 & 0 & 0 & 0 \\
0 & 0 & 0 & 0 & 0 & 0 & 0 & 0 & -\frac{\rho A \omega^{2} a}{i k_{1}} & \frac{\rho A \omega^{2}}{i k_{1}} & -\frac{\rho A \omega^{2} b}{i k_{2}} & \frac{\rho A \omega^{2}}{i k_{2}} \\
0 & 0 & 0 & 0 & 0 & 0 & 0 & 0 & -E I i k_{1} \alpha_{1} a & -E I i k_{1} \alpha_{1} & -E I i k_{2} \alpha_{2} b & -E I i k_{2} \alpha_{2}
\end{array}\right] .
$$

\section{Conflict of Interests}

The authors declare that there is no conflict of interests regarding the publication of this paper.

\section{Acknowledgments}

The authors would like to extend their thanks to the joint financial support by the National Natural Science Fund of China (51278215), the National Basic Research Program of China (973 Program: 2011CB013800), and the Fundamental Research Funds for the Central Universities (HUST: 2014QN211).

\section{References}

[1] K. K.-H. Tseng and A. S. K. Naidu, "Non-parametric damage detection and characterization using smart piezoceramic material," Smart Materials and Structures, vol. 11, no. 3, pp. 317-329, 2002.

[2] G. Park, H. Sohn, C. R. Farrar, and D. J. Inman, "Overview of piezoelectric impedance-based health monitoring and path forward," Shock and Vibration Digest, vol. 35, no. 6, pp. 451-463, 2003.

[3] G. Park, A. C. Rutherford, H. Sohn, and C. R. Farrar, "An outlier analysis framework for impedance-based structural health monitoring," Journal of Sound and Vibration, vol. 286, no. 1-2, pp. 229-250, 2005.

[4] V. Giurgiutiu and A. Zagrai, "Electro-mechanical impedance method for crack detection in metallic plates," in International Society for Optical Engineering, vol. 4335 of Proceedings of SPIE, pp. 131-142, Newport Beach, Calif, USA, 2001.

[5] V. Giurgiutiu and A. N. Zagrai, "Embedded self-sensing piezoelectric active sensors for on-line structural identification," Journal of Vibration and Acoustics, Transactions of the ASME, vol. 124, no. 1, pp. 116-125, 2002.

[6] V. Giurgiutiu and A. Zagrai, "Damage detection in thin plates and aerospace structures with the electro-mechanical impedance method," Structural Health Monitoring, vol. 4, no. 2, pp. 99-118, 2005.
[7] B. S. Divsholi and Y. Yang, "Health monitoring of steel structures using sub-frequency electromechanical impedance technique," Journal of Nondestructive Evaluation, vol. 31, no. 3, pp. 197-207, 2012.

[8] J. Min, S. Park, C. Yun, C. Lee, and C. Lee, "Impedance-based structural health monitoring incorporating neural network technique for identification of damage type and severity," Engineering Structures, vol. 39, pp. 210-220, 2012.

[9] D. S. Wang, H. Y. Song, and H. P. Zhu, "Numerical and experimental studies on damage detection of a concrete beam based on PZT admittances and correlation coefficient," Construction and Building Materials, vol. 49, pp. 564-574, 2013.

[10] H. Song, H. J. Lim, and H. Sohn, "Electromechanical impedance measurement from large structures using a dual piezoelectric transducer," Journal of Sound and Vibration, vol. 332, no. 25, pp. 6580-6595, 2013.

[11] S. Talakokula, S. Bhalla, and A. Gupta, "Corrosion assessment of reinforced concrete structures based on equivalent structural parameters using electro-mechanical impedance techniq," Journal of Intelligent Material Systems and Structures, vol. 25, no. 4, pp. 484-500, 2014.

[12] C. Liang, F. P. Sun, and C. A. Rogers, "Coupled electromechanical analysis of adaptive material systemsdetermination of the actuator power consumption and system energy transfer," Journal of Intelligent Material Systems and Structures, vol. 5, no. 1, pp. 12-20, 1994.

[13] S. Zhou, C. Liang, and C. A. Rogers, "An impedance-based system modeling approach for induced strain actuator-driven structures," Journal of Vibration and Acoustics, Transactions of the ASME, vol. 118, no. 3, pp. 323-331, 1996.

[14] S. Bhalla and C. K. Soh, "Structural health monitoring by piezoimpedance transducers. I: modeling," Journal of Aerospace Engineering, vol. 17, no. 4, pp. 154-165, 2004.

[15] V. G. M. Annamdas and C. K. Soh, "Three-dimensional electromechanical impedance model I: formulation of directional sum impedance," Journal of Aerospace Engineering, vol. 20, no. 1, pp. 53-62, 2007.

[16] V. G. M. Annamdas and C. K. Soh, "Three-dimensional electromechanical impedance model for multiple piezoceramic transducers-structure interaction," Journal of Aerospace Engineering, vol. 21, no. 1, pp. 35-44, 2008. 
[17] D. S. Wang, H. Y. Song, and H. P. Zhu, "Embedded 3D electromechanical impedance model for strength monitoring of concrete using a PZT transducer," Smart Materials and Structures, vol. 23, no. 11, Article ID 115019, 14 pages, 2014.

[18] J. F. Doyle, Wave Propagation in Structures, Springer, New York, NY, USA, 2nd edition, 1997.

[19] M. Palacz and M. Krawczuk, "Analysis of longitudinal wave propagation in a cracked rod by the spectral element method," Computers and Structures, vol. 80, no. 24, pp. 1809-1816, 2002.

[20] M. Krawczuk, M. Palacz, and W. Ostachowicz, "Wave propagation in plate structures for crack detection," Finite Elements in Analysis and Design, vol. 40, no. 9-10, pp. 991-1004, 2004.

[21] W. M. Ostachowicz, "Damage detection of structures using spectral finite element method," Computers and Structures, vol. 86, no. 3-5, pp. 454-462, 2008.

[22] P. Kudela, M. Krawczuk, and W. Ostachowicz, "Wave propagation modelling in 1D structures using spectral finite elements," Journal of Sound and Vibration, vol. 300, no. 1-2, pp. 88-100, 2007.

[23] A. Zak, M. Radzieński, M. Krawczuk, and W. Ostachowicz, "Damage detection strategies based on propagation of guided elastic waves," Smart Materials and Structures, vol. 21, no. 3, Article ID 035024, 2012.

[24] D. Samaratunga, R. Jha, and S. Gopalakrishnan, "Wavelet spectral finite element for wave propagation in shear deformable laminated composite plates," Composite Structures, vol. 108, pp. 341-353, 2013.

[25] J. Choi and D. J. Inman, "Spectrally formulated modeling of a cable-harnessed structure," Journal of Sound and Vibration, vol. 333, no. 14, pp. 3286-3304, 2014.

[26] G. Park, H. H. Cudney, and D. J. Inman, "An integrated health monitoring technique using structural impedance sensors," Journal of Intelligent Material Systems and Structures, vol. 11, no. 6, pp. 448-455, 2000.

[27] D. M. Peairs, D. J. Inman, and G. Park, "Circuit analysis of impedance-based health monitoring of beams using spectral elements," Structural Health Monitoring, vol. 6, no. 1, pp. 81-94, 2007.

[28] S. Ritdumrongkul, M. Abe, Y. Fujino, and T. Miyashita, "Quantitative health monitoring of bolted joints using a piezoceramic actuator-sensor," Smart Materials and Structures, vol. 13, no. 1, pp. 20-29, 2004.

[29] X. Wang and J. Tang, "Damage identification using piezoelectric impedance approach and spectral element method," Journal of Intelligent Material Systems and Structures, vol. 20, no. 8, pp. 907-921, 2009.

[30] Z. Guo and Z. Sun, "Impedance-based damage identification: a numerical study using spectral elements," in Proceedings of the Sensors and Smart Structures Technologies for Civil, Mechanical, and Aerospace Systems, San Diego, CA, USA, March 2010.

[31] W. Ostachowicz, P. Kudela, P. Malinowski et al., "Structural Health Monitoring based on piezoelectric sensors," in Proceedings of the 6th European Congress on Computational Methods in Applied Sciences and Engineering (ECCOMAS '12), pp. 64046413, Vienna, Austria, September 2012.

[32] J. Kim and K. W. Wang, "Impedance-based damage identification enhancement via tunable piezoelectric circuitry," in The International Society for Optical Engineering, vol. 9061 of Proceedings of SPIE, San Diego, Calif, USA, 2014.

[33] A. D. Dimarogonas and S. A. Paipetis, Analytical Methods in Rotor Dynamics, Applied Science Publishers, London, UK, 1983. 


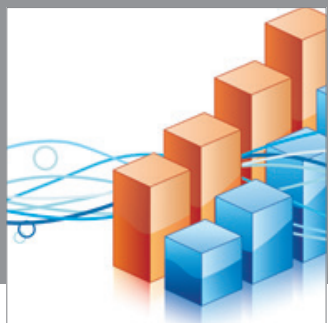

Advances in

Operations Research

mansans

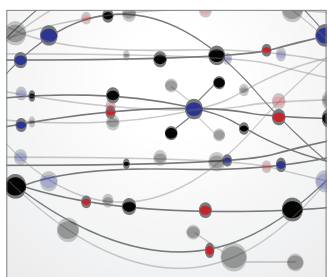

The Scientific World Journal
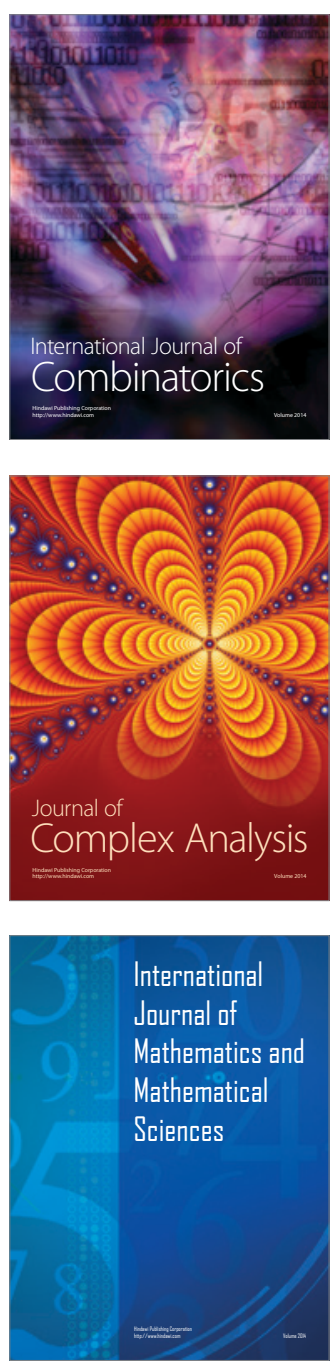
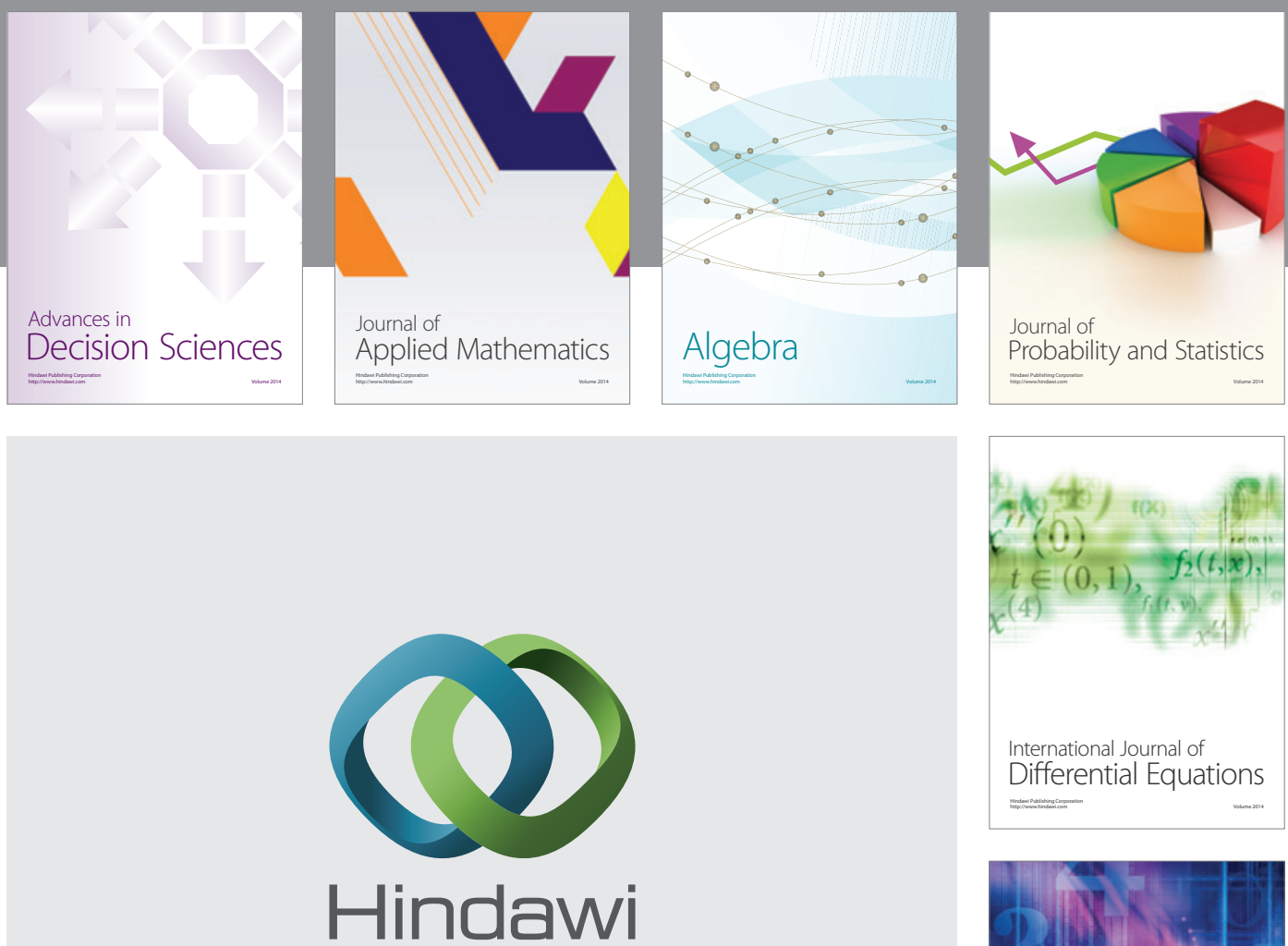

Submit your manuscripts at http://www.hindawi.com
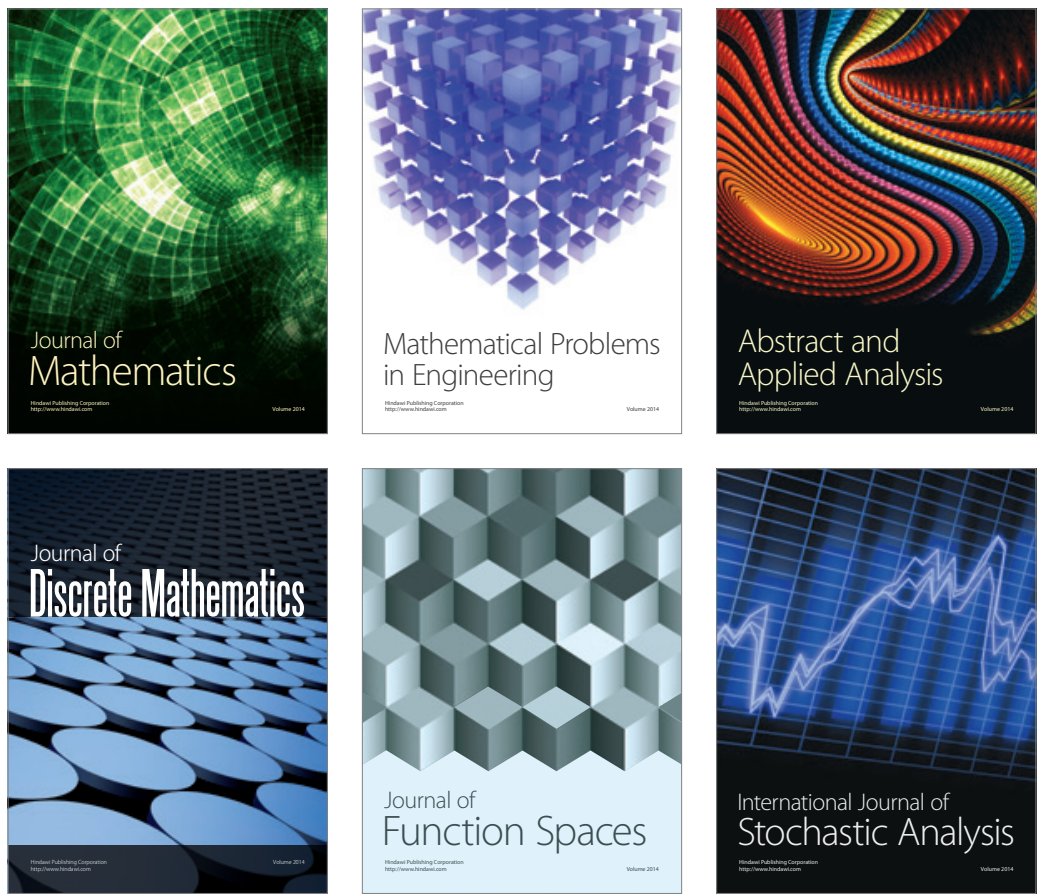

Journal of

Function Spaces

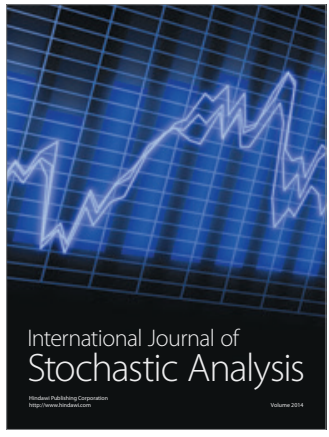

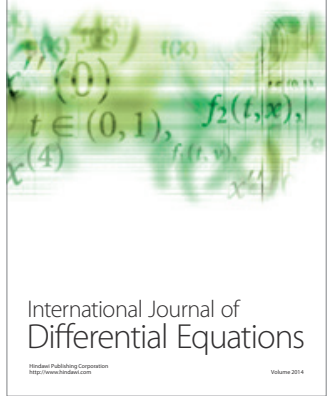
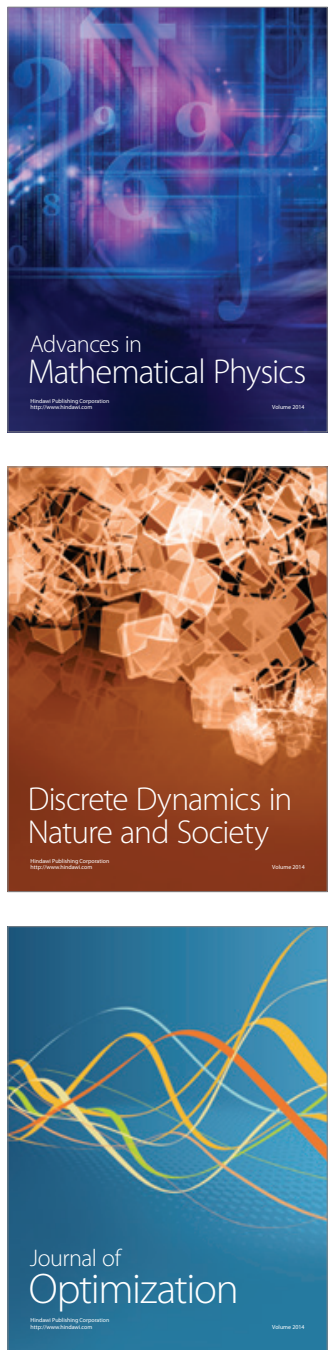